\title{
Heat shock protein 70 could enhance porcine epidemic diarrhoea virus replication by interacting with membrane proteins
}

\author{
Jae-Yeon Park', Jihoon Ryu ${ }^{2}$, Jung-Eun Park ${ }^{1,2}$, Eui-Ju Hong ${ }^{1,2}$ and Hyun-Jin Shin ${ }^{1,2^{*}}$ (0)
}

\begin{abstract}
In this study, we investigated the role of heat shock protein 70 (HSP70) in porcine epidemic diarrhoea virus (PEDV) replication. We found that PEDV infection induced strong HSP70 overexpression in the very early stage of infection. We also confirmed that HSP70 overexpression increased the speed of PEDV replication, resulting in the generation of more virions. In contrast, knockout of HSP70 in cells significantly downregulated PEDV protein expression, resulting in a significant reduction in PEDV replication. Most importantly, we confirmed that among the structural proteins of PEDV, membrane (M) proteins have this important role. We found that membrane proteins control cellular HSP70 expression in PEDV-infected cells. We confirmed HSP70/M complex formation by both immunoprecipitation and immunofluorescence assays. Additionally, PEDV M overexpression induced strong HSP70 expression. All our results clearly confirmed that in PEDV-infected cells, the M protein plays a very important role in PEDV replication in collaboration with HSP70.
\end{abstract}

Keywords: Porcine epidemic diarrhoea virus, heat shock protein 7, replication

\section{Introduction}

Viral replication is dependent on host cellular factors [1], several of which are necessary for viral replication [2]. In virus-infected cells, proteotoxic stress is strongly induced by viral protein synthesis [3], which has a negative effect on viral replication [4]. Cells respond to stress through adaptive changes that either limit or repair damage, thereby preventing cell death [5]. Heat shock protein 70 (HSP70) is an important protein that promotes cellular homeostasis during metabolism [6]. HSP70, a member of the heat shock protein family, is involved in various cellular processes through its role as a molecular chaperone [7]. In particular, it helps refold proteins that are misfolded or aggregated due to cell stress [8]. HSP70 family

\footnotetext{
*Correspondence: shin0089@cnu.ac.kr

${ }^{1}$ College of Veterinary Medicine, Chungnam National University, Daejeon 13434, Republic of Korea
}

Full list of author information is available at the end of the article members contain two domains: the N-terminal ATPase domain and the C-terminal substrate-binding domain [9]. ATP hydrolysis induces a conformational change in HSP70, triggering substrate folding and release [10].

Interaction with chaperones, such as heat shock proteins, is important for viral replication [4, 11]. Interactions between viral proteins and heat shock proteins have been reported in several viruses, such as tombusvirus, reovirus, adenovirus, polyomavirus, dengue virus, and influenza virus. [12-15]. However, not all viruses interact with host heat shock proteins. For example, HIV-1 viral protein $\mathrm{R}$ and the influenza $\mathrm{A}$ virus ribonucleoprotein negatively interact with HSP70 $[16,17]$.

Porcine epidemic diarrhoea virus (PEDV) infects swine intestines, causing enteric diseases along with vomiting, diarrhoea, dehydration, and high mortality in suckling piglets $[18,19]$. PEDV is a positive single-stranded RNA virus with an $\sim 28$ kilobase genome containing a $5^{\prime}$ cap and a $3^{\prime}$ polyadenylated tail. The genome comprises 
seven open reading frames (ORFs), including large polyproteins ORF1a, ORF1b, and ORF2-6, which encode 16 nonstructural proteins and four structural proteins [spike (S), envelope (E), membrane $(\mathrm{M})$, nucleocapsid $(\mathrm{N})]$, and accessory (ORF3) proteins [20,21]. The role of each structural protein has been well studied. The PEDV $\mathrm{N}$ protein interacts with nucleolar phosphoprotein nucleophosmin (NPM1), which promotes PEDV growth [22]. The authors noted that PEDV replicates in the cytoplasm, but the PEDV $\mathrm{N}$ protein is associated with nucleolar components other than NPM1. NPM1 overexpression induced PEDV replication, whereas NPM1 knockdown reduced PEDV replication. PEDV ORF3 interacts with cellular vacuolar protein sorting-associated protein 36 (VPS36) to inhibit PEDV replication [23].

The PEDV N protein prolongs the S-phase of the cell cycle, induces endoplasmic reticulum stress (ER stress), and upregulates interleukin-8 (IL-8) expression. PEDV ORF3 prolongs $\mathrm{S}$-phase and facilitates the formation of vesicles that promote the proliferation of PEDV [24]. PEDV ORF3 induces viral replication by inhibiting apoptosis in the host [25] and causes ER stress, facilitating autophagy [26]. In addition, the PEDV E protein causes ER stress and upregulates IL-8 expression. The PEDV $S$ protein is the main receptor-binding protein that interacts with host cell receptors [27]. There have been many studies on virulence factors in PEDV [28-30], but there are few reports on their correlation with host factors. In this study, we confirmed that HSP70 is important for PEDV replication, viral protein synthesis, and assembly.

\section{Materials and methods}

\section{Cells and viruses}

African green monkey kidney cell lines (Vero) were cultured in Minimum Essential Media (MEM) supplemented with $10 \mathrm{mM}$ HEPES (Gibco, USA) and 10\% antibiotic-antimycotic (Gibco, USA) foetal bovine serum (Gibco, USA) at $37{ }^{\circ} \mathrm{C}$ with $5 \% \mathrm{CO}_{2} .239 \mathrm{~T}$ cell lines were cultured in Dulbecco's modified Eagle's medium (DMEM) supplemented with $1 \times$ antibiotic-antimycotic and $10 \%$ foetal bovine serum at $37^{\circ} \mathrm{C}$ with $5 \% \mathrm{CO}_{2}$. IPEC J2 cell lines were cultured in Dulbecco's Modified Eagle Medium: Nutrient Mixture F-12 (DMEM/F12) supplemented with $1 \times$ Insulin-Transferrin-Selenium-G Supplement $(100 \times), 1 \times$ Antibiotic-Antimycotic, $10 \%$ foetal bovine serum, at $37{ }^{\circ} \mathrm{C}$ with $5 \% \mathrm{CO}_{2}$. In this study, we used the PEDV strain PED-CUP-B2014 [31]. Viral titres were determined by $\mathrm{TCID}_{50}$ and plaque assays [32].

\section{Plasmids}

The pCAGGS PEDV structure protein plasmid encoding the PEDV E, M, N and S proteins with a c-Myc fusion at its $\mathrm{C}$-terminus was constructed with the pCAGGS vector.
The cDNA for the PED-CUP-B2014. cDNA corresponding to the PEDV structure protein coding region was amplified with specific primers (Table 1). The pCAGGS HSP70 plasmid encoding Chlorocebus sabaeus HSP70 (NCBI Reference Sequence: XM_007973082.1) with a flag fusion at its $\mathrm{C}$-terminus was constructed by the pCAGGS vector. The pLenti-HSP70 plasmid encoding the HSP70.

\section{Antibodies}

Anti-HSP70 antibody (Enzo Life Science, ADI-SPA811-D), anti-c-Myc antibody (Santa Cruz, sc-40, sc-789), anti-flag antibody (Santa Cruz, sc-807) anti- $\beta$-actin (Santa Cruz, sc-47778) and mouse anti-PEDV were made in our laboratory (immunized inactivated PEDV in mouse).

\section{Heat shock and quercetin treatment to Vero cells}

To increase the expression of endogenous HSP70, Vero cells were heated at $45{ }^{\circ} \mathrm{C}$ for $20 \mathrm{~min}$ in a heating block and then transferred to a $37{ }^{\circ} \mathrm{C} \mathrm{CO}_{2}$ incubator. Vero cells were treated with $100 \mu \mathrm{M}$ quercetin (dissolved in DMSO and made a stock solution at $100 \mathrm{mM}$ ) for $12 \mathrm{~h}$ and then washed three times with PBS. In a preliminary study, $100 \mu \mathrm{M}$ quercetin was the best concentration in VERO cells after $12 \mathrm{~h}$ of incubation and no more activity afterwards (data not shown).

\section{Real-time PCR assay}

Vero cells were seeded in 6-well plates. The cells were washed with PBS and lysed in RiboEX Total RNA

\section{Table 1 Primer used in this study.}

\begin{tabular}{|c|c|}
\hline Primer & Sequence $\left(5^{\prime}-3^{\prime}\right)$ \\
\hline PEDVEF & CTCGAGGCCACCATGCTACAATTAGTGAATGATAATG \\
\hline PEDVER & AGATCTTACGTCAATAACAGTACTGGGGAGGG \\
\hline PEDV M F & CTCGAGGCCACCATGTCTAACGGTTCTATTCCCGTTG \\
\hline PEDV M R & AGATCTGACTAAATGAAGCACTTTCTCACTATC \\
\hline PEDV N F & CTCGAGGCCACCATGGCTTCTGTCAGCTTTCAGGATC \\
\hline PEDV N R & GGATCCATTTCCTGTATCGAAGATCTCGTTG \\
\hline PEDV SF & CTCGAGGCCACCATGAAGTCTTTAAATTACTTCTGG \\
\hline PEDV SR & GGTACCCTGCACGTGGACCTTTTCAAAAAC \\
\hline HSP70 F & GATCTCGCCACCATGGCCAAAGCCGCGGCGATC \\
\hline HSP70 R & CTCGAGATCTACCTCCTCAATGGTGGGGC \\
\hline qHSP70 F & AACGCCCTGGAGTCATACGC \\
\hline qHSP70 R & TCTTCTTGTCGGCCTCGCTG \\
\hline qPEDV M F & CGTACAGGTAAGTCAATTAC \\
\hline qPEDV M R & GATGAAGCATTGACTGAA \\
\hline qGAPDH F & CCTTCCGTGTCCCCACTGCCAAC \\
\hline qGAPDH R & GACGCCTGCTTCACCACCTTCT \\
\hline
\end{tabular}

Kozak sequence is underlined. Start codon is bolded. Italic sequence denotes restriction enzyme site. 
(GeneAll, Korea), and reverse transcription was performed using BioFACT ${ }^{\mathrm{TM}} 2 \times \mathrm{RT}^{\mathrm{R}}$ Pre-Mix (BioFACT $^{\mathrm{TM}}$, Korea) amplified with random hexamer (Thermo Fisher, USA). Amplification was carried out in a $20 \mu \mathrm{L}$ reaction mixture containing $10 \mu \mathrm{L}_{\text {TOPreal }}{ }^{\mathrm{TM}}$ qPCR $2 \times$ premix (Enzynomics, Korea), $0.2 \mu \mathrm{M}$ concentration of each primer (Table 1 ), and $1 \mu \mathrm{L}$ cDNA. The reaction procedure was $95^{\circ} \mathrm{C}$ for $5 \mathrm{~min}$, followed by 40 cycles at $95{ }^{\circ} \mathrm{C}$ for $30 \mathrm{~s}, 58{ }^{\circ} \mathrm{C} 30 \mathrm{~s}$ and $72{ }^{\circ} \mathrm{C} 30 \mathrm{~s}$. The relative mRNA expression level was normalized to the housekeeping gene GAPDH. The relative transcript levels were analysed using the $\Delta \Delta \mathrm{Ct}$ method.

\section{Confocal fluorescence microscopy}

We followed the general manual for confocal microscopy. Briefly, Vero cells were seeded in 12-well plates. The cells were treated with PED-CUP-2014B $(\mathrm{MOI}=1)$. At $18 \mathrm{~h}$ post-infection, the cells were fixed with $4 \%$ paraformaldehyde for $15 \mathrm{~min}$. Following fixation three times washed in PBS, the cells were then permeabilized with $0.25 \%$ Triton X-100 for 10 min and blocked in PBS containing $2 \%$ BSA for $1 \mathrm{~h}$ at room temperature. After three washes with PBS, the coverslips were incubated with primary antibodies in PBS containing $2 \%$ BSA at $4{ }^{\circ} \mathrm{C}$ (overnight). After being washed with PBS, the coverslips were incubated with Alexa Fluor 488 (Invitrogen, A32723) and Alexa Fluor 594 (Invitrogen, A32740) for $2 \mathrm{~h}$ at room temperature. After washing with PBS, the nuclei were stained on Hoechst 33258 (Thermo Fisher, 62249). After staining for $15 \mathrm{~min}$, the cells were washed with PBS and mounted onto microscope slides. Fluorescence signals were observed under confocal microscopy. Transfected samples were also subjected to the same procedure.

\section{Western blots}

The cells were washed with cold PBS and lysed in RIPA buffer (Thermo Fisher, USA) containing protease inhibitor cocktail (Sigma Aldrich, Germany) for $30 \mathrm{~min}$ at $4{ }^{\circ} \mathrm{C}$, and then the supernatant was collected. The protein concentration was determined with a BCA protein assay kit (Thermo Fisher, USA). SDS-PAGE and Western blotting were carried out using standard methods. Briefly, equivalent amounts of protein were separated on polyacrylamide-tricine gels (8-12\% polyacrylamide). After SDS-PAGE, the gels were transferred onto $0.45 \mu \mathrm{m}$ polyvinylidene fluoride (PVDF) membranes (Millipore, USA) followed by blocking with 5\% BSA in TBST (TBS with $0.1 \%$ Tween 20 ) for $1 \mathrm{~h}$ at room temperature. The membrane was incubated with primary antibody at $4{ }^{\circ} \mathrm{C}$ overnight. After being washed with TBST, the membrane was incubated with HRP-tagged anti-rabbit IgG and anti-mouse IgG (1:10 000 dilution) for $2 \mathrm{~h}$ at room temperature. The images were observed with ECL solution
(SuperSignal West Femto Maximum Sensitivity Substrate 34095) using an ATTO Luminograph (Japan).

\section{Immunoprecipitation assay}

293 T cells were seeded in 6-well plates. Cells were transfected with $2 \mu \mathrm{g}$ of pCAGGS EGFP, PEDV E (envelope), PEDV $M$ (membrane), PEDV $N$ (nucleocapsid) and PEDV S (spike) plasmids using PEI (12332A, Invitrogen). At $48 \mathrm{~h}$ post-transfection, the cells were washed in cold PBS, lysed with RIPA buffer for $30 \mathrm{~min}$ at $4{ }^{\circ} \mathrm{C}$ with protease inhibitor (Thermo Fisher, 88265), and then the supernatant was collected. Anti-c-myc was added to each tube and incubated overnight at $4{ }^{\circ} \mathrm{C}$. Then, the AG beads (Santa Cruz, ab-2003) were incubated with the above samples overnight at $4{ }^{\circ} \mathrm{C}$. After washing four times with RIPA buffer, the eluted samples were separated by SDSPAGE on $12 \%$ gels under reducing conditions.

\section{Gene knockdown by siRNA}

siRNAs obtained from GenePharma (Shanghai, China) were designed to bind endogenous HSP70 (GenBank Accession Number XM_007973082). Vero cells were seeded into 12-well plates, and the transfection mixture was prepared with $200 \mu \mathrm{L}$ of Opti-MEM medium (Gibco, USA) containing Lipofectamine 3000 (Invitrogen, USA) and $200 \mu \mathrm{M}$ of each siRNA (Table 2). At $48 \mathrm{~h}$ post-transfection, the cells were prepared for various experiments. The silencing efficiency was determined by Western blot assay.

\section{Statistical analysis}

Data are presented as the mean \pm standard deviation (SD). Statistical significance was calculated using SPSS and GraphPad Prism 8. A $p$ value $<0.05$ was considered statistically significant. Asterisks in figures indicate statistical significance $\left(" p<0.05,{ }^{* * *} p<0.01,{ }^{* * * *} p<0.001\right)$.

\section{Results \\ PEDV infection significantly increased HSP70 expression} We investigated the correlation between PEDV infection and HSP70 expression. PEDV-infected Vero cells

Table 2 siRNA used in this study.

\begin{tabular}{ll}
\hline siRNA & Sequence $\left(\mathbf{5}^{\prime} \mathbf{- 3}^{\prime}\right)$ \\
\hline$\# 576 \mathrm{~F}$ & GCAACGUGCUCAUCUUUGATT \\
$\# 576 \mathrm{R}$ & UCAAAGAUGAGCACGUUGCTT \\
$\# 1070 \mathrm{~F}$ & GACCUGAACAAGAGCAUCATT \\
$\# 1070 \mathrm{R}$ & UGAUGCUCUUGUUCAGGUCTT \\
scramble F & UUCUCCGAACGUGUCACGUTT \\
scramble R & ACGUGACACGUUCGGAGAATT \\
\hline
\end{tabular}


induced significantly higher expression of HSP70 than mock-infected cells in a time-dependent manner (Figure 1A). We found that HSP70 expression was induced at $12 \mathrm{~h}$ post-infection (hpi), and its levels increased $\sim$ tenfold compared to the levels at $8 \mathrm{hpi}$. Shockingly, HSP70 expression increased by 10000 -fold between 12 and $24 \mathrm{hpi}$. Based on these results, we concluded that PEDV infection strongly upregulates HSP70 expression. We also examined PEDV mRNA levels over time. We found that PEDV mRNA levels significantly increased from 8 hpi onwards, reaching a maximum at 24 hpi, which closely mirrored HSP70 activation (Figure 1B). We detected PEDV mRNA at 8 hpi and identified an $\sim 70 \%$ increase between 12 and $18 \mathrm{hpi}$. These results were confirmed by Western blot. Western blot analysis confirmed that endogenous HSP70 expression was induced from 8 hpi $(\sim 2.5$-fold higher than mock control), and there was a large increase between 8 and 12 hpi (Figure 1C). In addition, HSP70 expression increased by $\sim 3.5$-fold by $24 \mathrm{hpi}$. Taken together, our results clearly demonstrate that PEDV infection strongly upregulates endogenous HSP70 mRNA and protein expression. Additionally, we wanted to confirm our results in other PEDV-susceptible cell lines to ensure that the results were not specific to Vero cells. Vero cells are of monkey origin; thus, we wanted to confirm our findings in a swine cell line. We performed similar experiments using IPEC-J2 cells. Western blot analysis revealed similar findings in IPEC-J2 and Vero cells in relation to PEDV infection (Figure 1E). PEDV induced endogenous HSP70 expression in IPEC-J2 cells in an MOI-dependent manner. PEDV infection increased endogenous HSP70 expression levels by $\sim 30-50 \%$ compared to mock-infected cells (Figure $1 \mathrm{~F}$ ).
A

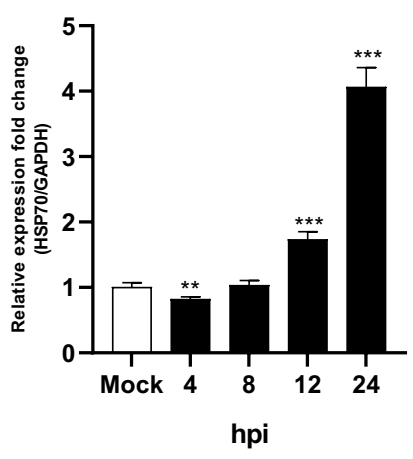

D

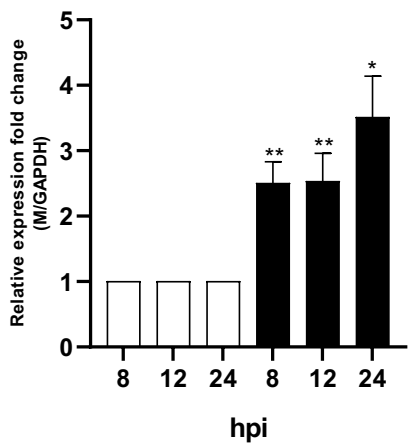

B

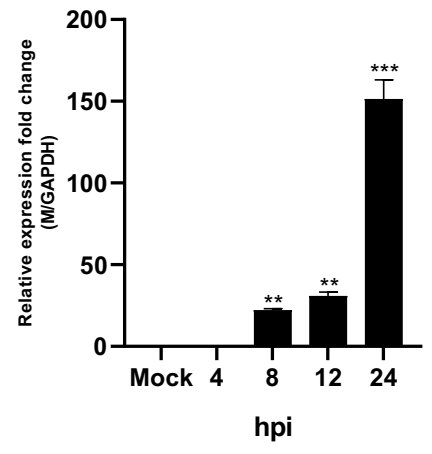

E

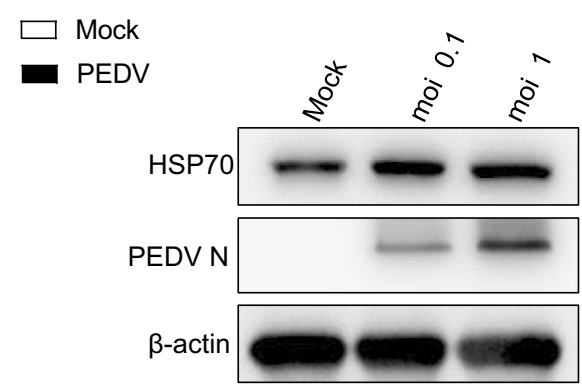

C

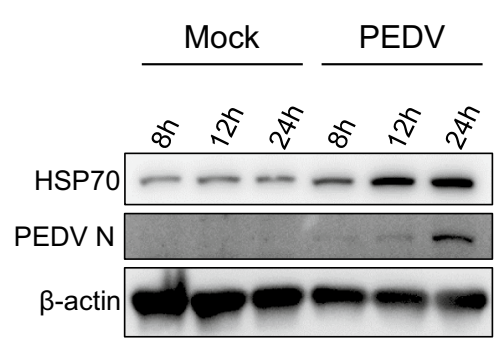

$\mathbf{F}$

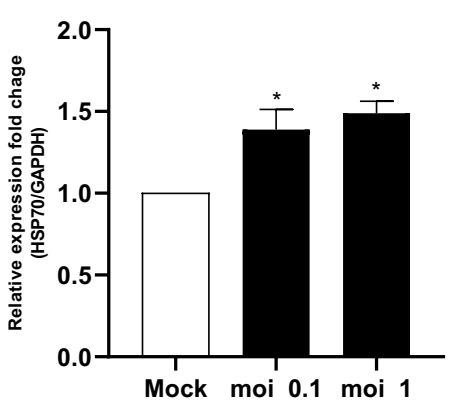

Figure 1 Cellular HSP70 expression level in PEDV-infected cells. A-B Vero cells were infected with PEDV at an MOI of 1 and harvested at different times as indicated. Total RNA was isolated to analyse HSP70 and viral M gene mRNA levels by quantitative RT-PCR. The mRNA levels of HSP70 and viral M were normalized to the mRNA levels of GAPDH. C-D Cell extracts were analysed by Western blot using anti-HSP70, anti-PEDV, and anti- $\beta$-actin antibodies. $\beta$-actin served as an internal control. The protein levels of HSP70 and PEDV were quantified by determining band intensities and normalized to $\beta$-actin. E IPEC-J2 cells were infected with PEDV at an MOI of 0.1 or 1. Cells were harvested at 24 hpi. The cell extracts were analysed by Western blot using anti-HSP70, anti-PEDV, and anti- $\beta$-actin antibodies. $\beta$-actin served as an internal control. F The levels of HSP70 and PEDV protein in IPEC-J2 cells were quantified by determining band intensities, and subsequent normalization to the levels of data is the mean \pm SD $\left(n=3,{ }^{*} p<0.05,{ }^{* *} p<0.01,{ }^{* * *} p<0.001\right)$. 


\section{Colocalization of PEDV and HSP70}

Colocalization of HSP70 and PEDV was confirmed using confocal microscopy. In PEDV-infected Vero cells, PEDV was found only in the cytoplasm (Figure 2E). We confirmed the results using anti-PEDV $\mathrm{N}$ protein antibodies generated in mice. As shown in Figure 2D, HSP70 was also highly expressed in almost the same location as the PEDV protein. When we merged, PEDV proteins were expressed in exactly the same locations where HSP70 was expressed (Figure 2F). Therefore, we concluded that HSP70 and PEDV colocalized in PEDV-infected Vero cells.

\section{Conformation of correlation using HSP70 inhibitors and heat shock treatment}

Next, to confirm the role of HSP70 in PEDV replication, we used the HSP inhibitor quercetin as an HSP synthesis inhibitor and heat shock as an HSP70 activator. We compared HSP70 expression at 12 and $24 \mathrm{~h}$ after treatment. As shown in the Western blot data, quercetin treatment inhibited PEDV protein expression by $27 \%$ ( 0.77 and 1.00 , respectively) at $12 \mathrm{~h}$ (Figures $3 \mathrm{~A}$ and $\mathrm{C}$ ). In contrast, heat shock treatment $\left(45{ }^{\circ} \mathrm{C}\right)$ resulted in a $31 \%$ increase in PEDV protein expression (1.00 and 1.31, respectively). However, there were no differences associated with

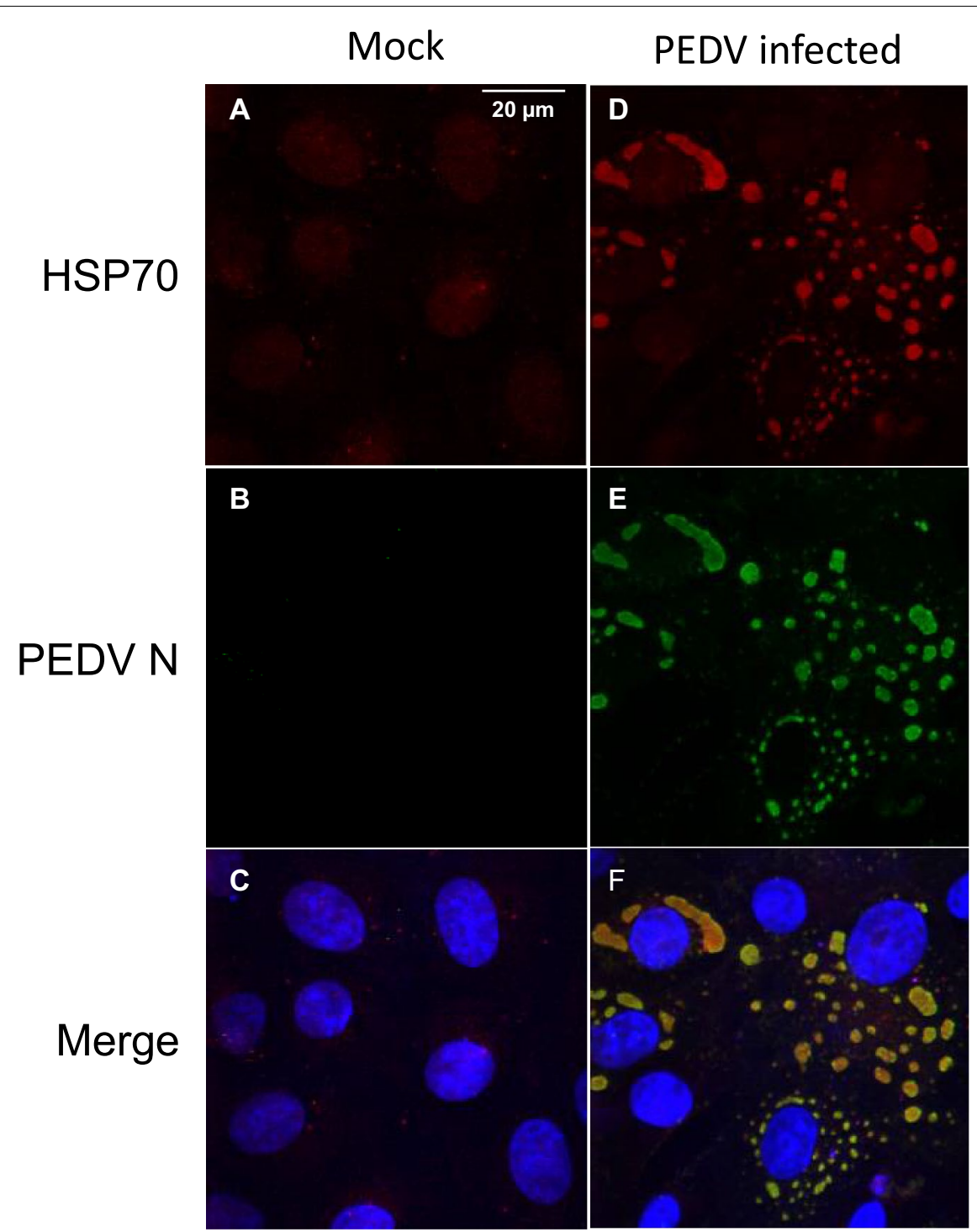

Figure 2 Colocalization of HSP70 and PEDV. Vero cells were infected with PEDV at an MOI of 1. Cells were fixed at 24 hpi, and double immunofluorescence analysis was performed with anti-PEDV (green) and anti-HSP70 (red) antibodies. Mock treatments served as negative controls. The nuclei were stained with Hoechst 333258 (blue). A Anti- HSP70 staining of mock-infected cells. B Anti-PEDV staining of mock-infected cells. C Merged image. D Anti- HSP70 staining of PEDV-infected cells. E Anti-PEDV staining of PEDV-infected cells. F Merged image. 
A

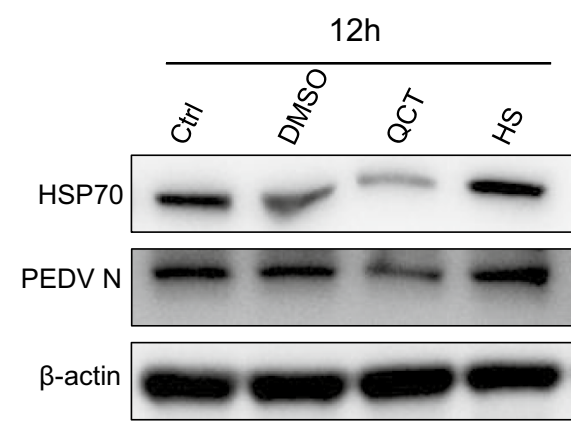

C

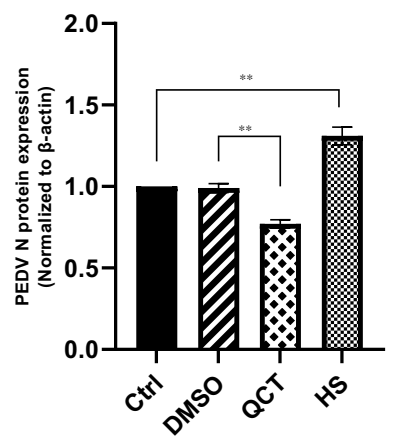

B

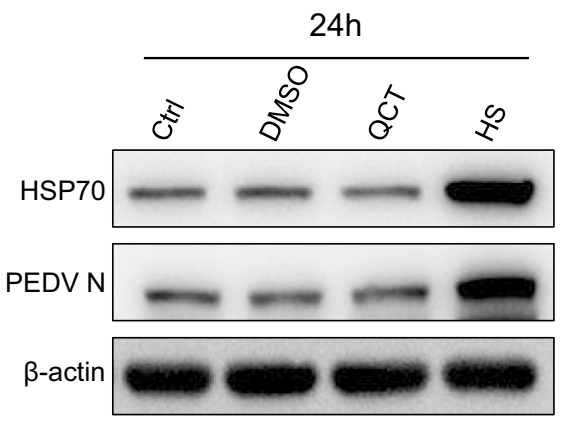

D

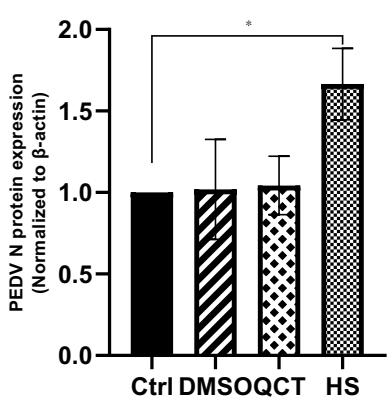

Figure 3 Quercetin and heat shock treatment. A-D Vero cells were heated (HS) or not (Ctrl) or treated with quercetin (100 $\mu$ M; QCT) or not (DMSO). Twelve hours later, cells were inoculated with PEDV at an MOI of 1. Cells were harvested at $12 \mathrm{~h}$ and $24 \mathrm{~h}$ for Western blot analysis. The levels of PEDV N protein were quantified by determining band intensities, which were then normalized to the levels of $\beta$-actin. Data are the mean $\pm \mathrm{SD}\left(n=3,{ }^{*} p<0.05,{ }^{* *} p<0.01,{ }^{* * *} p<0.001\right)$ A Cell lysates collected at $12 \mathrm{hpi}$ were subjected to Western blot assay with anti-HSP70, anti-PEDV, and anti- $\beta$-actin antibodies. B Cell lysates collected at $24 \mathrm{hpi}$ were subjected to Western blot assay with anti-HSP70, anti-PEDV, and anti- $\beta$-actin antibodies. C-D Quantification of $12 \mathrm{~h}$ and $24 \mathrm{~h}$ results.

quercetin treatment at $24 \mathrm{~h}(1.00$ and 1.04) (Figures 3B and $\mathrm{D})$. These results are similar to our preliminary data demonstrating that quercetin worked maximally at $12 \mathrm{~h}$ and had no effect afterwards (data not shown). In contrast, heat shock treatment increased HSP70 expression by approximately $67 \%$ at $24 \mathrm{~h}$ ( 1.00 and 1.67 , respectively) (Figure 3B).

\section{Viral titre changes induced by HSP70 and heat shock treatment}

We also compared viral replication by measuring viral titers after quercetin and heat shock treatment. There was a $22 \%$ decrease in PEDV titre following $12 \mathrm{~h}$ of quercetin treatment ( $\mathrm{TCID}_{50} 2.5$ and 1.95 , respectively) (Figure 4A). In contrast, there was an $18 \%$ increase in PEDV titre following $12 \mathrm{~h}$ of heat shock treatment $\left(\mathrm{TCID}_{50} 2.5\right.$ and 2.99 , respectively). However, there was no difference between the quercetin, DMSO, and negative control groups at $24 \mathrm{~h}\left(\mathrm{TCID}_{50} 4.25,4.30\right.$, and 4.30 , respectively) (Figure 4B). However, there was still a 9.4\% increase in PEDV titre following $24 \mathrm{~h}$ of heat shock treatment $\left(\mathrm{TCID}_{50} 4.25\right.$ and 4.65 , respectively). PEDV protein levels also increased by $\sim 2.5$-fold following heat shock treatment but decreased by $\sim 50 \%$ following quercetin treatment (Figure 4C). Therefore, we concluded that HSP70 expression significantly increased PEDV protein expression and resulted in the production of more progeny virions.

\section{The effect of knockdown or overexpression of HSP70 on PEDV replication}

To confirm our findings, we performed HSP70 knockdown using siRNAs. Among the siRNAs tested, siRNA576 demonstrated the best results, and siRNA1070 produced reasonable results. In all knockdown experiments, scrambled siRNA was used as a negative control. The results showed that knockdown of HSP70 using siRNAs significantly reduced PEDV protein synthesis 
A

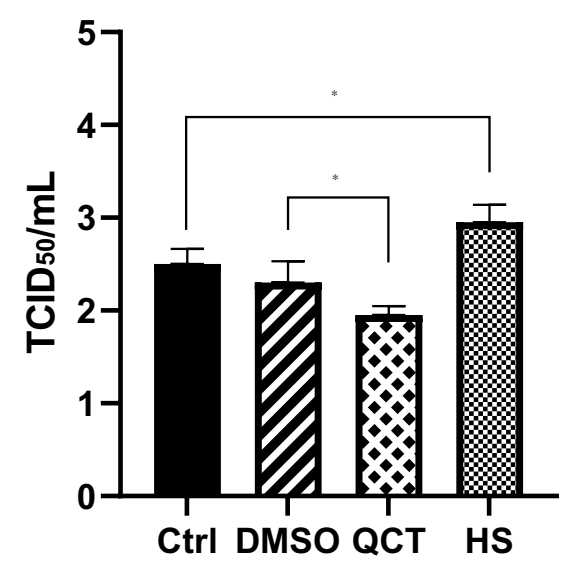

B

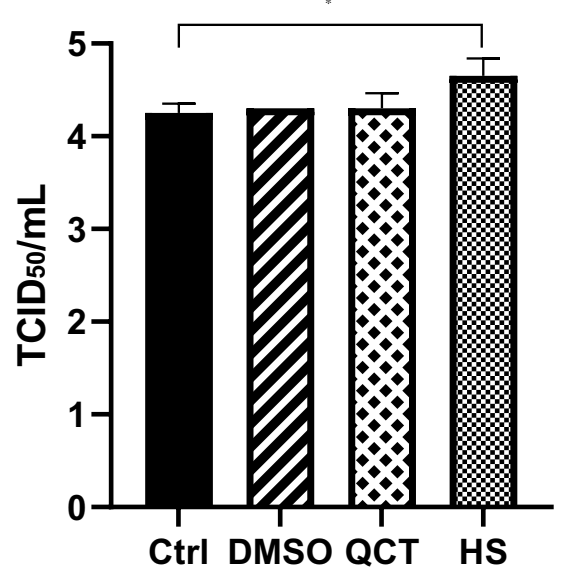

Figure 4 The effects of quercetin and heat shock on viral titre. Vero cells were heated (HS) or not (Ctrl) or treated with quercetin (100 $\mu M$; QCT) or not (DMSO). Twelve hours later, cells were inoculated with PEDV at an $\mathrm{MOI}$ of 1 . The viral titers of cell supernatants were determined at 12 hpi and $24 \mathrm{hpi}$. Data are mean $\pm \operatorname{SD}\left(n=3,{ }^{*} p<0.05\right)$. A Viral titre after $12 \mathrm{~h}$. B Viral titre after $24 \mathrm{~h}$.

(Figure 5A). HSP70 knockdown resulted in an $\sim 70 \%$ reduction in PEDV titre with siRNA576 and a $25 \%$ reduction with siRNA1070 (Figure 5B). No change was observed in the negative control. We then examined the Western blot results (Figure 5A) and found that these results were similar to what was observed in an HSP70stable cell line. The results showed that PEDV protein expression was high in the HSP70-stable cell line (Figures $5 \mathrm{C}-\mathrm{E})$. These results strongly suggest that PEDV protein synthesis is closely related to HSP70 expression.

\section{The correlation between PEDV structural proteins and HSP70}

Next, we sought to determine which PEDV structural proteins are correlated with HSP70. We transfected each of the PEDV structural proteins (E, M, N and S) and performed immunoprecipitation (IP). As shown in Figure 6A, the PEDV M protein clearly immunoprecipitated with HSP70. PEDV $M$ protein expressed in two forms, one in stacking gel (marked as 2 in Figure) and the other as approximately $28 \mathrm{kDa}$ (marked as 1 in Figure). As shown by the IP results, only the PEDV $M$ protein showed significant results, although there were very minor bands from the other structural proteins. As an expression control, we also showed whole-cell lysate images. To confirm this hypothesis, we performed IP experiments only with the $M$ protein and found that the $M$ protein clearly immunoprecipitated with HSP70, as shown in Figure 6B. Similar to Figure 6A, immunoprecipitated PEDV M (number 1) was located at $28 \mathrm{kDa}$, and PEDV $M$ (number 2) was located in stacking gels in the input data.

\section{Colocalization of the PEDV M protein and HSP70}

Colocalization of the PEDV $M$ protein and HSP70 was confirmed using confocal microscopy. As shown by the IFA results, only the PEDV $M$ protein clearly colocalized with HSP70 (Figure 7D). We could not detect colocalization of HSP70 with any PEDV structural proteins other than the $M$ protein. Even though there were very minor bands in the IPs with other structural proteins (Figure 6A), confocal microscopy clearly confirmed the colocalization of HSP70 and the PEDV M protein. Based on these results, we concluded that the PEDV $M$ protein directly interacts with HSP70, and this interaction activates both HSP70 expression and PEDV replication.

\section{Specificity of the correlation between the PEDV M protein and HSP70}

Next, we examined the specificity of the correlation between HSP70 and the PEDV M protein. First, we monitored changes in HSP70 expression in response to various amounts of the PEDV $M$ protein. We transfected different amounts $(1 \mu \mathrm{g}$ and $2 \mu \mathrm{g})$ of the $\mathrm{M}$ protein plasmid pCAGGS-PEDV-M together with either HSP70 or EGFP plasmids. In Western blot results, transfection of $2 \mu \mathrm{g}$ of PEDV M DNA resulted in higher HSP70 expression than in cells transfected with only $1 \mu \mathrm{g}$ (Figure 8A). Actin-normalized data revealed that PEDV $M$ expression increased $\sim$ threefold compared to the empty vector-transfected control (Figure 8A). Interestingly, in the same EGFP overexpression experiments, even with increased PEDV $M$, there was no change in the level of EGFP expression (Figure $8 \mathrm{~B}$ ). Based on these results, we concluded that PEDV 
A

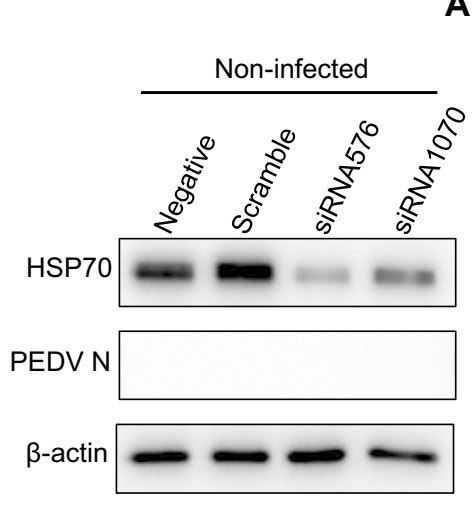

C

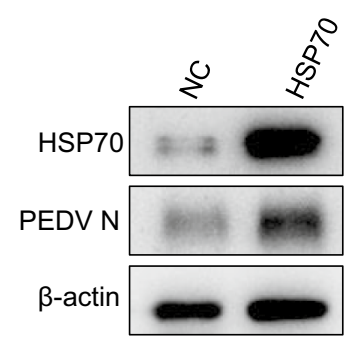

D

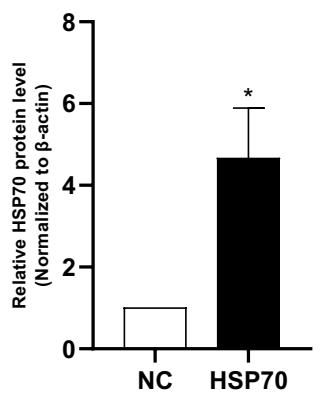

B
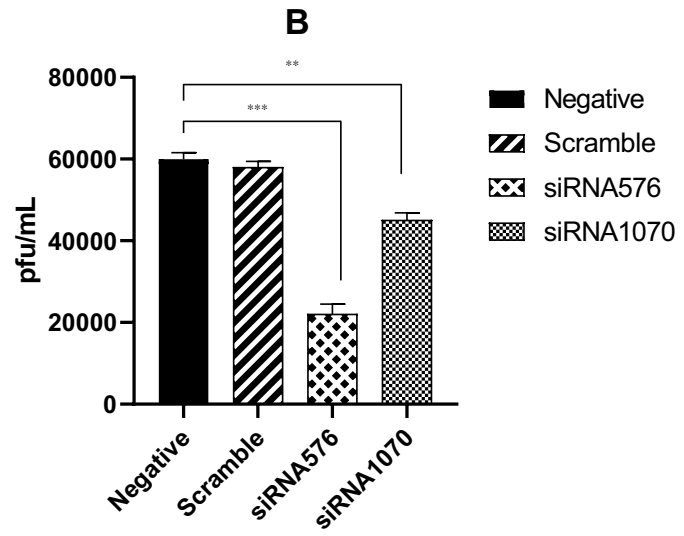

E

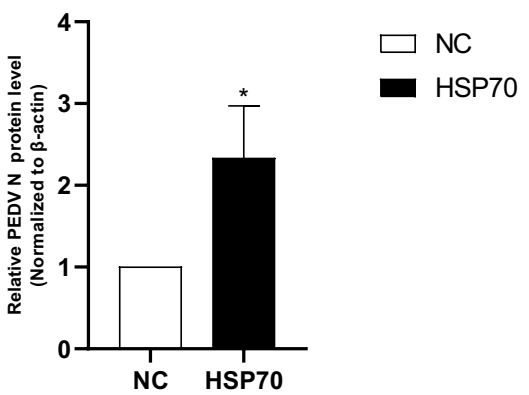

Figure 5 Comparison of HSP70 and PEDV protein expression in HSP70 knockdown and overexpressed cells. Vero cells were transfected with no siRNA (negative), scramble siRNA (scramble), or different siRNAs targeting HSP70 (siRNA576 and siRNA1070). Twenty-four hours later, the cells were mock-infected or infected with PEDV at an MOI of 1. A Western blot analysis of noninfected and PEDV-infected cells. B The viral titers of cell supernatants were determined $24 \mathrm{hpi}$. Data are mean $\pm \mathrm{SD}(n=3)$. C Vero cells were infected with a lentivirus control (NC) or pLenti-HSP70 (HSP70). Cells were harvested at 24 hpi for Western blot analysis. D-E The levels of HSP70 and PEDV protein were quantified by determining band intensities and subsequent normalization to the levels of $\beta$-actin. Data are the mean $\pm \operatorname{SD}\left(n=3,{ }^{*} p<0.05,{ }^{* *} p<0.01,{ }^{* * *} p<0.001\right)$.

M specifically interacts with HSP70 in a dose-dependent manner.

\section{Discussion}

Many positive-stranded RNA viruses produce specific proteins that interact with chaperones in infected cells. The role of these proteins relates to the folding of abundant viral proteins, regulation of viral replication via activation of replication proteins, and reduction of host antiviral responses. Among these host chaperones, HSP70 has been reported to be most frequently involved in viral replication. Changes in HSP70 expression induced by viral infection control intracellular defence mechanisms. For example, in influenza A virus, the ribonucleoprotein is interrupted by HSP70, which blocks its replication in vitro and in vivo [17]. HIV-1 viral protein $R$ (Vpr) is related to G2 cell cycle arrest. HSP70 counteracts Vpr G2 growth arrest, which is positively regulated by HIV-1 replication [16]. Rotavirus (RF strain) viral protein synthesis and progeny virus production were increased by HSP70 silencing in Caco-2 cells. HSP70 silencing reduced the ubiquitination of rotavirus structural proteins [33]. Many viruses utilize HSP70 for their replication. In porcine reproductive and respiratory syndrome virus, upregulation of HSP70 in MARC-145 cells significantly increased viral protein levels and virion production. In classical swine fever virus, NS5A directly interacts with HSP70 and promotes HCV replication $[34,35]$. In classical swine fever virus, NS5A directly interacts with HSP70, an interaction that plays a pivotal role in the viral life cycle [36]. In tomato bushy stunt virus, the replicase complex requires HSP70, which plays a role in the assembly of viral replicase [37]. In dengue virus infection, downregulation of HSP70 in THP-1 cells significantly decreased viral protein and virion production levels [38]. Kong et al. reported that HSP70 was upregulated in the intestine in both cold temperatureexposed pigs and cold-exposed Vero cells. They concluded that HSP70 positively regulated PEDV mRNA 


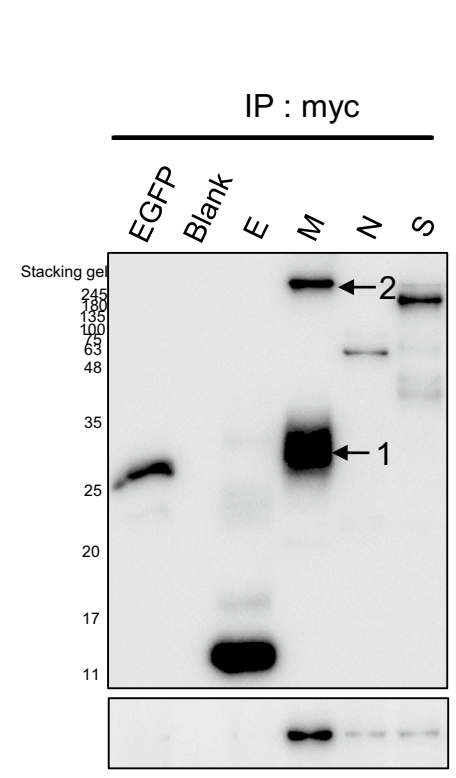

A

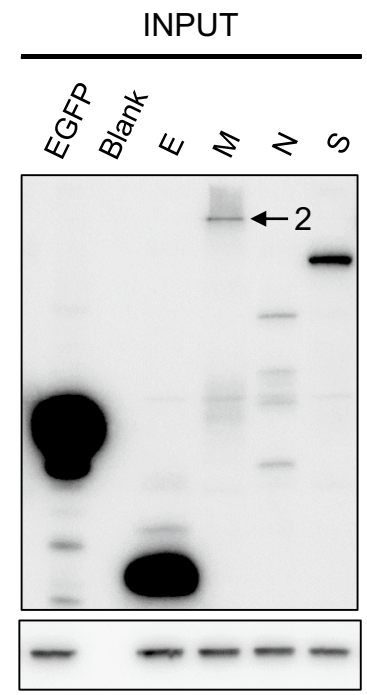

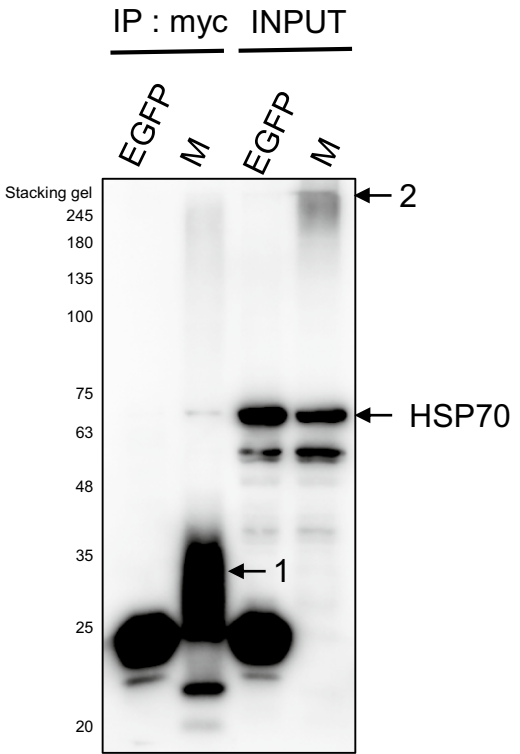

Figure 6 Interaction between the PEDV M protein and HSP70. Cells were transfected with PEDV structure proteins. After 48 h, the cells were harvested, and immunoprecipitation was performed. The transfected protein molecular weights were as follows: PEDV E (13 kDa), PEDV M (28 kDa), PEDV N (55 kDa) and PEDV S (150 kDa). A Immunoprecipitation assays demonstrated that endogenous HSP70 bound only to the PEDV M protein in transfected cells. PEDV M protein expressed in two forms, one in stacking gel (marked as 2 in Figure) and the other as approximately $28 \mathrm{kDa}$ (marked as 1 in Figure). B As a form of validation, cells were transfected with HSP70 and PEDV M protein DNA only, and the analysis was performed again. All immunoprecipitation data clearly confirmed that immunoprecipitated PEDV M (number 1) was located at 28 kDa, and PEDV M (number 2) was located in stacking gels in the input data.
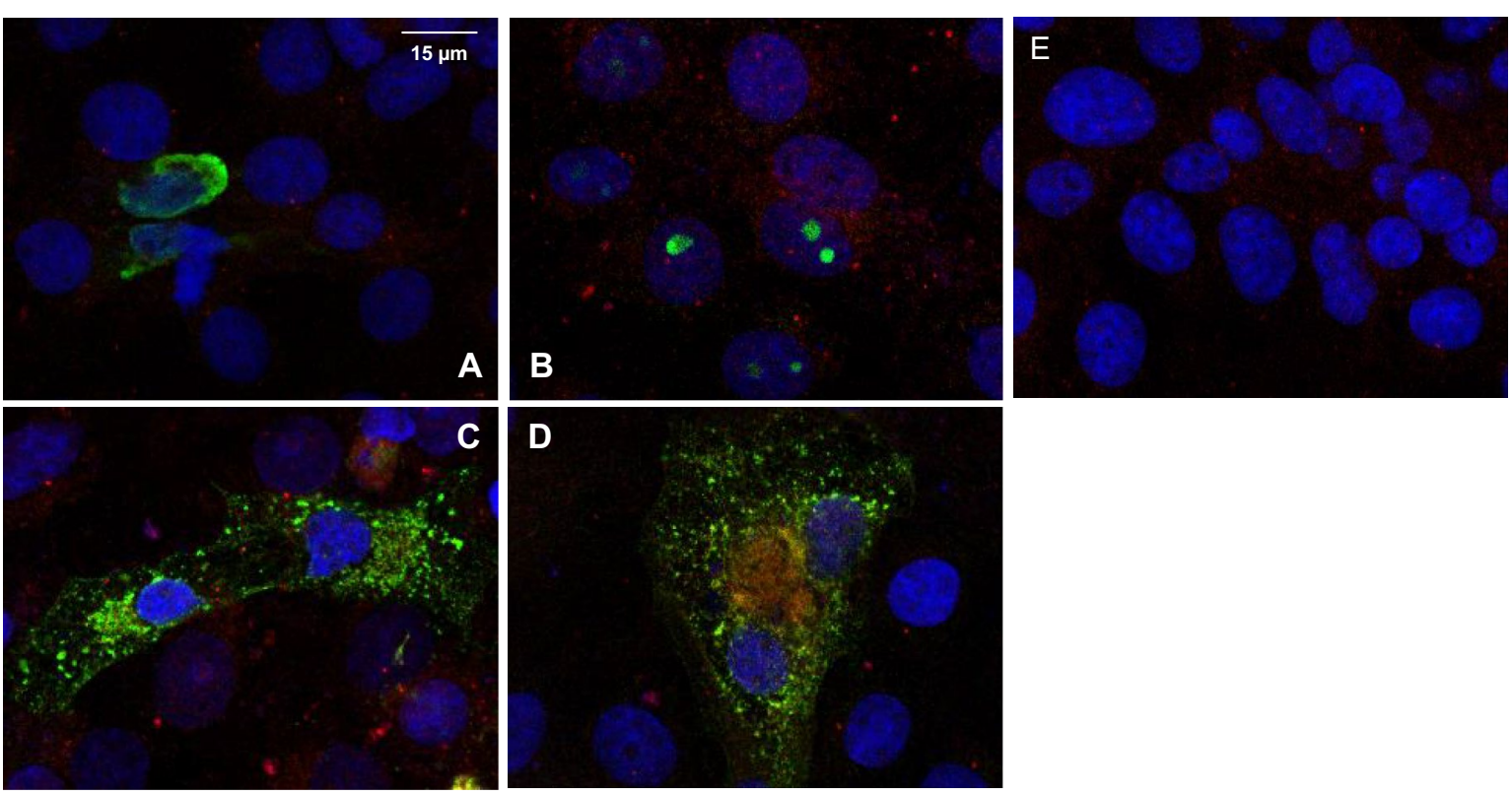

Figure 7 Colocalization of PEDV M and HSP70. Cells were transfected with PEDV structure proteins. After 48 h, IFA was performed with anti-Myc (green) and anti-HSP70 (red) antibodies. Nuclei were stained with Hoechst 33,258 (blue). Colocalization between PEDV M and HSP70 was confirmed by confocal microscopy. A pCAGGS PEDV N-myc, B pCAGGS PEDV S-myc, C pCAGGS PEDV E-myc, D pCAGGS PEDV M-myc, and E non-transfected control. All images are merged. 
A

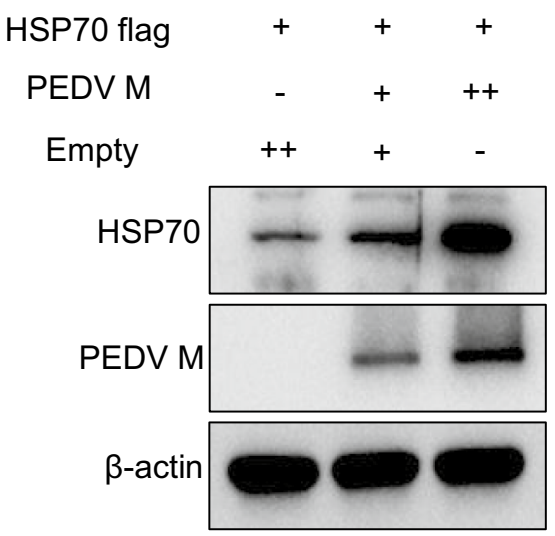

B

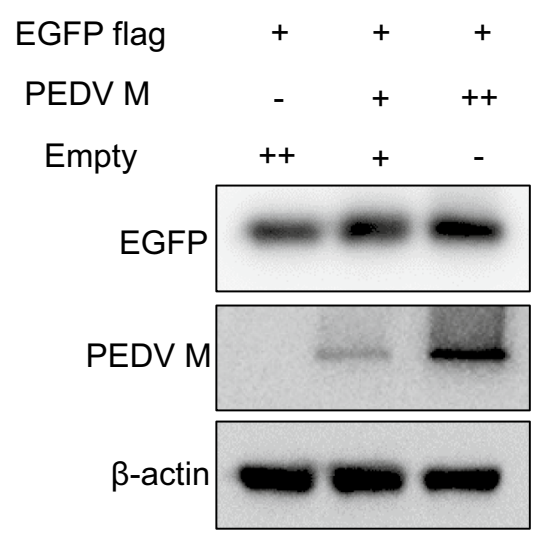

Figure 8 Specificity of the correlation between PEDV M and HSP70 expression. Cells were transfected with 1 or $2 \mu g$ of PEDV M DNA together with either HSP70 or EGFP plasmids. After 48 h, cells were harvested and subjected to Western blot analysis. A Cells were cotransfected with 1 or $2 \mu \mathrm{g}$ of PEDV M DNA together with HSP70 and subjected to Western blot analysis. B Cells were cotransfected with 1 or $2 \mu \mathrm{g}$ of PEDV M DNA together with EGFP and subjected to Western blot analysis.

synthesis and protein expression. These results are similar to our results in the correlation between HSP70 and PEDV replication [39].

In the present study, we examined the correlation between PEDV and HSP70. We found that HSP70 plays a very important role in PEDV replication. PEDV infection induced the expression of HSP70, and as a result, PEDV replicated in high numbers and eventually generated much higher progeny virions (Figure 1). As expected, heat shock treatment induced higher levels of viral proteins and viral replication. In contrast, quercetin treatment significantly decreased the levels of viral proteins and viral replication (Figures 3 and 4). Knockdown of HSP70 by siRNAs clearly confirmed that inhibition of HSP70 reduces PEDV protein expression and viral replication (Figures 5A and B). Conversely, overexpression of HSP70 increased PEDV protein expression and viral replication (Figures $5 \mathrm{C}$ and D). In conclusion, HSP70 plays a very important role in PEDV replication. Although we were not able to completely block HSP70 expression (too many cellular HSP70 molecules in many different cellular locations), our results are still meaningful.

The PEDV $M$ protein is a membrane protein that is wrapped around viral particles. Previous studies have identified that the PEDV $M$ protein plays an important role in viral assembly [40]. Additionally, the PEDV M protein affects cell growth, cell cycle progression, and interleukin 8 (IL-8) expression. Expression of the PEDV $M$ protein in an intestinal epithelial cell line arrested the cell cycle at S-phase via the cyclin
A pathway. However, the PEDV M protein does not induce endoplasmic reticulum stress and does not activate NF- $\mathrm{KB}$ [41]. In addition to functioning in virion formation and cell cycle arrest, the PEDV $M$ protein also reduces host cell immune responses. Recent LCMS/MS studies have reported several potential binding partners of the PEDV M protein [42].

In this study, we detected a direct interaction between the PEDV M protein and HSP70. We found that PEDV $\mathrm{M}$ and HSP70 colocalized and coimmunoprecipitated (Figures 6 and 7). This correlation between $M$ and HSP70 occurred in a dose-dependent manner (Figure 8). It was not Vero cell specific because the correlation was also confirmed in IPEC-J2 cells (Figure 1).

Further studies examining the interaction between HSP70 and non-structural PEDV proteins are required. In summary, we demonstrated that HSP70 plays a very important role in PEDV replication. PEDV infection induced HSP70 expression, which boosted PEDV protein expression and the production of progeny virions. While the PEDV M protein directly interacts with HSP70 and promotes PEDV replication, the mechanism through which HSP70 promotes PEDV replication is unclear. Further mechanism study is needed.

\section{Acknowledgements}

This research was supported by a grant from the Technology Development Program for Agriculture and Forestry, Ministry for Food, Agriculture, Forestry and Fisheries. This research was supported by a grant from the Technology Development Program for Agriculture and Forestry, Ministry for Food, Agriculture, Forestry and Fisheries. 


\section{Authors' contributions}

JYP and JHR designed experiments and collected data. HJS supervised the project, analysed the data and prepared the manuscript. JEP and EJH provided technical advice. All authors read and approved the final manuscript.

\section{Declarations}

\section{Competing interests}

The authors declare that they have no competing interests.

\section{Author details}

${ }^{1}$ College of Veterinary Medicine, Chungnam National University, Daejeon 13434, Republic of Korea. ${ }^{2}$ Research Institute of Veterinary Medicine, Chungnam National University, Daejeon 13434, Republic of Korea.

Received: 21 June 2021 Accepted: 14 September 2021

Published online: 30 October 2021

\section{References}

1. de Wilde AH, Snijder EJ, Kikkert M, van Hemert MJ (2018) Host factors in coronavirus replication. Curr Top Microbiol Immunol 419:1-42. https:// doi.org/10.1007/82_2017_25

2. Nagy PD, Pogany J (2011) The dependence of viral RNA replication on co-opted host factors. Nat Rev Microbiol 10:137-149. https://doi.org/10. 1038/nrmicro2692

3. Fung TS, Liao Y, Liu DX (2016) Regulation of stress responses and translational control by coronavirus. Viruses 8:184. https://doi.org/10.3390/ v8070184

4. Mayer MP (2005) Recruitment of Hsp70 chaperones: a crucial part of viral survival strategies. Rev Physiol Biochem Pharmacol 153:1-46. https://doi. org/10.1007/s10254-004-0025-5

5. Srivastava P (2002) Roles of heat-shock proteins in innate and adaptive immunity. Nat Rev Immunol 2:185-194. https://doi.org/10.1038/nri749

6. Young JC, Barral JM, Ulrich Hartl F (2003) More than folding: localized functions of cytosolic chaperones. Trends Biochem 28:541-547

7. Rosenzweig R, Nillegoda NB, Mayer MP, Bukau B (2019) The Hsp70 chaperone network. Nat Rev Mol Cell Biol 20:665-680. https://doi.org/10. 1038/s41580-019-0133-3

8. Hartl FU (1996) Molecular chaperones in cellular protein folding. Nature 381:571-579. https://doi.org/10.1038/381571a0

9. Heymann JB, Iwasaki K, Yim Y-I, Cheng N, Belnap DM, Greene LE, Eisenberg E, Steven AC (2005) Visualization of the binding of Hsc70 ATPase to clathrin baskets: implications for an uncoating mechanism. J Biol Chem 280:7156-7161. https://doi.org/10.1074/jbc.M411712200

10. Mayer MP, Bukau B (2005) Hsp70 chaperones: cellular functions and molecular mechanism. Cell Mol Life Sci 62:670-684. https://doi.org/10. 1007/s00018-004-4464-6

11. Santoro MG (2000) Heat shock factors and the control of the stress response. Biochem Pharmacol 59:55-63

12. Chromy LR, Pipas JM, Garcea RL (2003) Chaperone-mediated in vitro assembly of polyomavirus capsids. Proc Natl Acad Sci USA 100:1047710482. https://doi.org/10.1073/pnas.1832245100

13. Glotzer JB, Saltik M, Chiocca S, Michou Al, Moseley P, Cotten M (2000) Activation of heat-shock response by an adenovirus is essential for virus replication. Nature 407:207-211. https://doi.org/10.1038/35025102

14. Ivanovic T, Agosto MA, Chandran K, Nibert ML (2007) A role for molecular chaperone Hsc70 in reovirus outer capsid disassembly. J Biol Chem 282:12210-12219. https://doi.org/10.1074/jbc.M610258200

15. Wang RYL, Stork J, Nagy PD (2009) A key role for heat shock protein 70 in the localization and insertion of tombusvirus replication proteins to intracellular membranes. J Virol 83:3276-3287. https://doi.org/10.1128/ Jvi.02313-08

16. Iordanskiy S, Zhao Y, Dubrovsky L, lordanskaya T, Chen M, Liang D, Bukrinsky M (2004) Heat shock protein 70 protects cells from cell cycle arrest and apoptosis induced by human immunodeficiency virus type 1 viral protein R. J Virol 78:9697-9704. https://doi.org/10.1128/JVI.78.18.96979704.2004
17. Li G, Zhang J, Tong X, Liu W, Ye X (2011) Heat shock protein 70 inhibits the activity of Influenza A virus ribonucleoprotein and blocks the replication of virus in vitro and in vivo. PLoS ONE 6:e16546. https://doi.org/10.1371/ journal.pone.0016546

18. Li W, van Kuppeveld FJM, He Q, Rottier PJM, Bosch BJ (2016) Cellular entry of the porcine epidemic diarrhea virus. Virus Res 226:117-127. https://doi. org/10.1016/j.virusres.2016.05.031

19. Song D, Park B (2012) Porcine epidemic diarrhoea virus: a comprehensive review of molecular epidemiology, diagnosis, and vaccines. Virus Genes 44:167-175. https://doi.org/10.1007/s11262-012-0713-1

20. Kocherhans R, Bridgen A, Ackermann M, Tobler K (2001) Completion of the porcine epidemic diarrhoea coronavirus (PEDV) genome sequence. Virus Genes 23:137-144. https://doi.org/10.1023/a:1011831902219

21. Wang K, Lu W, Chen J, Xie S, Shi H, Hsu H, Yu W, Xu K, Bian C, Fischer WB, Schwarz W, Feng L, Sun B (2012) PEDV ORF3 encodes an ion channel protein and regulates virus production. FEBS Lett 586:384-391. https:// doi.org/10.1016/j.febslet.2012.01.005

22. Shi D, Shi H, Sun D, Chen J, Zhang X, Wang X, Zhang J, Ji Z, Liu J, Cao L, Zhu X, Yuan J, Dong H, Wang X, Chang T, Liu Y, Feng L (2017) Nucleocapsid interacts with NPM1 and protects it from proteolytic cleavage, enhancing cell survival, and is involved in PEDV growth. Sci Rep 7:39700. https://doi.org/10.1038/srep39700

23. Kaewborisuth C, Yingchutrakul Y, Roytrakul S, Jongkaewwattana A (2019) Porcine Epidemic Diarrhea Virus (PEDV) ORF3 interactome reveals inhibition of virus replication by cellular VPS36 protein. Viruses 11:382. https:// doi.org/10.3390/v11040382

24. Ye S, Li Z, Chen F, Li W, Guo X, Hu H, He Q (2015) Porcine epidemic diarrhea virus ORF3 gene prolongs S-phase, facilitates formation of vesicles and promotes the proliferation of attenuated PEDV. Virus Genes 51:385-392. https://doi.org/10.1007/s11262-015-1257-y

25. Si F, Hu X, Wang C, Chen B, Wang R, Dong S, Yu R, Li Z (2020) Porcine epidemic diarrhea virus (PEDV) ORF3 enhances viral proliferation by inhibiting apoptosis of infected cells. Viruses 12:214. https://doi.org/10. 3390/v12020214

26. Zou D, Xu J, Duan $X, X u X$, Li P, Cheng L, Zheng L, Li X, Zhang Y, Wang $X$, Wu X, Shen Y, Yao X, Wei J, Yao L, Li L, Song B, Ma J, Liu X, Wu Z, Zhang $\mathrm{H}$, Cao H (2019) Porcine epidemic diarrhea virus ORF3 protein causes endoplasmic reticulum stress to facilitate autophagy. Vet Microbiol 235:209-219. https://doi.org/10.1016/j.vetmic.2019.07.005

27. Wicht O, Li W, Willems L, Meuleman TJ, Wubbolts RW, van Kuppeveld FJ, Rottier PJ, Bosch BJ (2014) Proteolytic activation of the porcine epidemic diarrhea coronavirus spike fusion protein by trypsin in cell culture. J Virol 88:7952-7961. https://doi.org/10.1128/JVI.00297-14

28. Liu C, Tang J, Ma Y, Liang X, Yang Y, Peng G, Qi Q, Jiang S, Li J, Du L, Li F (2015) Receptor usage and cell entry of porcine epidemic diarrhea coronavirus. J Virol 89:6121-6125. https://doi.org/10.1128/JVI.00430-15

29. Li BX, Ge JW, Li YJ (2007) Porcine aminopeptidase N is a functional receptor for the PEDV coronavirus. Virology 365:166-172. https://doi.org/10. 1016/j.virol.2007.03.031

30. Meng FD, Suo SQGW, Zarlenga DS, Cong YY, Ma XW, Zhao Q, Ren XF (2014) A phage-displayed peptide recognizing porcine aminopeptidase $\mathrm{N}$ is a potent small molecule inhibitor of PEDV entry. Virology 456:20-27. https://doi.org/10.1016/j.virol.2014.01.010

31. Park JE, Kang KJ, Ryu JH, Park JY, Jang H, Sung DJ, Kang JG, Shin HJ (2018) Porcine epidemic diarrhea vaccine evaluation using a newly isolated strain from Korea. Vet Microbiol 221:19-26. https://doi.org/10.1016/j. vetmic.2018.05.012

32. Cruz DJ, Shin HJ (2007) Application of a focus formation assay for detection and titration of porcine epidemic diarrhea virus. J Virol Methods 145:56-61. https://doi.org/10.1016/j.jviromet.2007.05.012

33. Broquet $A H$, Lenoir C, Gardet A, Sapin C, Chwetzoff S, Jouniaux AM, Lopez S, Trugnan G, Bachelet M, Thomas G (2007) Hsp70 negatively controls rotavirus protein bioavailability in caco- 2 cells infected by the rotavirus RF strain. J Virol 81:1297-1304. https://doi.org/10.1128/JVI.01336-06

34. Gonzalez O, Fontanes V, Raychaudhuri S, Loo R, Loo J, Arumugaswami V, Sun R, Dasgupta A, French SW (2009) The heat shock protein inhibitor quercetin attenuates hepatitis C virus production. Hepatology 50:17561764. https://doi.org/10.1002/hep.23232

35. Khachatoorian R, Ganapathy E, Ahmadieh Y, Wheatley N, Sundberg C, Jung CL, Arumugaswami V, Raychaudhuri S, Dasgupta A, French SW (2014) The NS5A-binding heat shock proteins HSC70 and HSP70 play 
distinct roles in the hepatitis C viral life cycle. Virology 454-455:118-127. https://doi.org/10.1016/j.virol.2014.02.016

36. Zhang CC, Kang K, Ning PB, Peng YX, Lin Z, Cui HJ, Cao Z, Wang J, Zhang YM (2015) Heat shock protein 70 is associated with CSFV NS5A protein and enhances viral RNA replication. Virology 482:9-18. https://doi.org/10. 1016/j.virol.2015.02.014

37. Pogany J, Stork J, Li Z, Nagy PD (2008) In vitro assembly of the Tomato bushy stunt virus replicase requires the host Heat shock protein 70. Proc Natl Acad Sci USA 105:19956-19961. https://doi.org/10.1073/pnas.08108 51105

38. Padwad YS, Mishra KP, Jain M, Chanda S, Ganju L (2010) Dengue virus infection activates cellular chaperone Hsp70 in THP-1 cells: downregulation of Hsp70 by siRNA revealed decreased viral replication. Viral Immunol 23:557-565. https://doi.org/10.1089/vim.2010.0052

39. Kong F, Xu Y, Ran W, Yin B, Feng L, Sun D (2020) Cold exposure-induced up-regulation of Hsp70 positively regulates PEDV mRNA synthesis and protein expression in vitro. Pathogens 9:246. https://doi.org/10.3390/ pathogens 9040246
40. Arndt AL, Larson BJ, Hogue BG (2010) A conserved domain in the coronavirus membrane protein tail is important for virus assembly. J Virol 84:11418-11428. https://doi.org/10.1128/Jvi.01131-10

41. Xu XG, Zhang HL, Zhang Q, Dong J, Huang Y, Tong DW (2015) Porcine epidemic diarrhea virus $\mathrm{M}$ protein blocks cell cycle progression at S-phase and its subcellular localization in the porcine intestinal epithelial cells. Acta Virol 59:265-275. https://doi.org/10.4149/av_2015_03_265

42. Wang RY, Yu RS, Chen BQ, Si FS, Wang J, Xie CF, Men CF, Dong SJ, Li Z (2020) Identification of host cell proteins that interact with the M protein of porcine epidemic diarrhea virus. Vet Microbiol 246:108729. https://doi. org/10.1016/j.vetmic.2020.108729

\section{Publisher's Note}

Springer Nature remains neutral with regard to jurisdictional claims in published maps and institutional affiliations.
Ready to submit your research? Choose BMC and benefit from:

- fast, convenient online submission

- thorough peer review by experienced researchers in your field

- rapid publication on acceptance

- support for research data, including large and complex data types

- gold Open Access which fosters wider collaboration and increased citations

- maximum visibility for your research: over $100 \mathrm{M}$ website views per year

At $\mathrm{BMC}$, research is always in progress.

Learn more biomedcentral.com/submissions 